\title{
The Efficacy and Safety of Eravacycline in the Treatment of Complicated Intra-Abdominal Infections: A Systemic Review and Meta-Analysis of Randomized Controlled Trials
}

\author{
Shao-Huan Lan ${ }^{1}{ }^{(}$, Shen-Peng Chang ${ }^{2}$, Chih-Cheng Lai ${ }^{3}{ }^{\circledR}$, Li-Chin Lu $^{4}$ \\ and Chien-Ming Chao ${ }^{3, *}$ \\ 1 School of Pharmaceutical Sciences and Medical Technology, Putian University, Putian 351100, Fujian, China; \\ shawnlan0713@gmail.com \\ 2 Department of Pharmacy, Chi Mei Medical Center, Liouying 73657, Taiwan; httremoon@ms.szmc.edu.tw \\ 3 Department of Intensive Care Medicine, Chi Mei Medical Center, Liouying 73657, Taiwan; \\ dtmed141@gmail.com \\ 4 School of Management, Putian University, Putian 351100, China; jane90467@gmail.com \\ * Correspondence: ccm870958@yahoo.com.tw
}

Received: 27 May 2019; Accepted: 13 June 2019; Published: 17 June 2019

check for updates

\begin{abstract}
This study aims to assess the clinical efficacy and safety of eravacycline for treating complicated intra-abdominal infection (cIAI) in adult patients. The PubMed, Web of Science, EBSCO, Cochrane databases, Ovid Medline, Embase, and ClinicalTrials.gov were searched up to May 2019. Only randomized controlled trials (RCTs) that evaluated eravacycline and other comparators for the treatment of cIAI were included. The primary outcome was the clinical cure rate at the test-of-cure visit based on modified intent-to-treat population, microbiological intent-to-treat population, clinically evaluable population, and microbiological evaluable population, and the secondary outcomes were clinical failure rate and the risk of adverse event. Three RCTs were included. Overall, eravacycline had a clinical cure rate $(88.7 \%, 559 / 630)$ at test-of-cure in modified intent-to-treat population similar to comparators $(90.1 \%, 492 / 546)$ in the treatment of cIAIs (risk ratio (RR), 0.99; 95\% confidence interval (CI), 0.95-1.03; $I^{2}=0 \%$, Figure 3). In the microbiological intent-to-treat, clinically evaluable, and microbiological evaluable populations, no difference was found between eravacycline and comparators in terms of clinical cure rate at test-of-cure (microbiological intent-to-treat population, RR, 0.99; 95\% CI, 0.95-1.04; $I^{2}=0 \%$, clinically evaluable population, RR, $1.00 ; 95 \%$ CI, $0.97-1.03 ; I^{2}=0 \%$, microbiological evaluable population, RR, 0.98; 95\% CI, 0.95-1.02; $I^{2}=0 \%$ ). In addition, eravacycline had clinical failure rate similar to comparators at test-of-cure in modified intent-to-treat population (RR, $\left.1.01 ; 95 \% \mathrm{CI}, 0.61-0.69 ; I^{2}=0 \%\right)$, microbiological intent-to-treat population (RR, 1.34; 95\% CI, 0.77-2.31; $I^{2}=16 \%$ ), clinically evaluable population (RR, $1.03 ; 95 \% \mathrm{CI}, 0.61-1.76 ; I^{2}=0 \%$ ), and microbiological evaluable population ( $R R, 1.32 ; 95 \%$ CI, 0.75-2.32; $I^{2}=10 \%$ ). Although eravacycline was associated with higher risk of treatment-emergent adverse event than comparators (RR, 1.34; 95\% CI, 1.13-1.58; $\left.I^{2}=0 \%\right)$, no significant differences were found between eravacycline and comparators for the risk of serious adverse event (RR, 1.04; 95\% CI, 0.65-1.65; $\left.I^{2}=0 \%\right)$, discontinuation of study drug because of adverse event (RR, 0.68; 95\% CI, 0.23-1.99; $I^{2}=13 \%$ ), and all-cause mortality (RR, 1.09; 95\% CI, $0.41-2.9 ; I^{2}=28 \%$ ). In conclusion, the clinical efficacy of eravacycline is as high as that of the comparator drugs in the treatment of cIAIs and this antibiotic is as well tolerated as the comparators.
\end{abstract}

Keywords: eravacycline; complicated intra-abdominal infection; efficacy; safety; mortality 


\section{Introduction}

In contrast to uncomplicated abdominal infections, complicated intra-abdominal infections (cIAIs) can extend beyond the originally infected organ into peritoneal spaces, and can be associated with local or diffuse peritonitis [1,2]. Enterobacteriaceae, especially Escherichia coli and Klebsiella pneumoniae, are the most common pathogens causing cIAIs [3-5]. Emergence of multiple antibiotic resistances has become the major concern in this clinical entity and further limits the choice of optimal antibiotic treatment. E. coli, Proteus species, and K. pneumoniae are the most common pathogens; however, high resistance to broad-spectrum antibiotics, including extended-spectrum $\beta$-lactams and fluoroquinolones, among these pathogens, also emerges as a critical threat worldwide.

Eravacycline is a novel, synthetic fluorocycline antibacterial agent [6], and has excellent bactericidal activity against most antibiotic-resistant pathogens according to several in vitro studies [7-10]. Recently, the clinical efficacy of eravacycline in cIAI has been evaluated in several clinical studies [11-13]. However, an updated meta-analysis comparing the efficacy and safety of eravacycline and other comparators for the treatment of cIAI is lacking. Therefore, we conducted this meta-analysis to provide real-time evidence about the efficacy and safety of cIAI.

\section{Methods}

\subsection{Study Search and Selection}

All clinical studies were identified through a systematic review of the literature in PubMed, Web of Science, EBSCO, Cochrane databases, Ovid Medline, Embase, and ClinicalTrials.gov until May 2019 using the following search terms: "eravacycline", "Xerava"TM", "TP-434", and "abdom*" (Search strategy presented in Appendix A). Studies were considered eligible for inclusion if they directly compared the clinical efficacy and safety of eravacycline with other antimicrobial agents in the treatment of adult patients with cIAIs. Studies were excluded if they focused on in vitro activity, animal studies, or pharmacokinetic-pharmacodynamic assessment. Two authors (S.-P.C. and S.-H.L.) searched and examined publications independently. When they disagreed, the third author (C.-C.L.) resolved the issue. The following data including year of publication, study design, type of infections, patients' demographic features, antimicrobial regimens, clinical and microbiological outcomes, and adverse effects were extracted from every included study.

\subsection{Outcome Measurement}

The primary outcome of this meta-analysis was clinical response assessed at the test-of-cure visit, end-of-treatment, and follow-up visit based on modified intent-to-treat population, microbiological intent-to-treat population, clinically evaluable population, microbiological evaluable populations. The intent-to-treat population included all randomized patients, and the modified intent-to-treat population included all intent-to treat patients who received any amount of study drug. The microbiological intent-to-treat population included all modified intent-to-treat patients who met the minimal disease definition of cIAI and had a baseline pathogen identified. The clinically evaluable population included all modified intent-to-treat patients who met the minimal disease definition of cIAI and had a clinical response assessed at the test-of-cure visit. The microbiological evaluable population included all clinically evaluable patients who had a baseline pathogen identified and a microbiological response assessed. Clinical response was classified as cure, failure, or indeterminate based on clinical outcomes. Clinical cure was defined as resolution of all or most pretherapy signs or symptoms with no further requirement for antibiotics, radiological intervention, or surgery. The safety population included all patients who received any intravenous study therapy. Treatment-emergent adverse events were defined as adverse events that started during or after the first dose of study drug administration or increased in severity or relationship to the study drugs during the study. Serious adverse event is defined as an untoward medical occurrence or effect that at any dose results in 
death, is life-threatening, requires hospitalization or extension of existing hospitalization, or results in persistent or significant disability.

\subsection{Data Analysis}

The quality of enrolled RCTs and the risk of bias were assessed using Cochrane Risk of Bias Assessment tool [14]. Statistical analyses were conducted using the software Review Manager, version 5.3. The degree of heterogeneity was evaluated with the $Q$ statistic generated from the $\chi^{2}$ test. The proportion of statistical heterogeneity was assessed using the $I^{2}$ measure. Heterogeneity was considered significant when the $p$ was less than 0.1 or $I^{2}$ was greater than $50 \%$. The random-effects model was used when data were significantly heterogeneous and the fixed-effect model was used when data were homogeneous. Pooled risk ratios (RRs) and 95\% confidence intervals (CIs) were calculated for outcome analyses.

\section{Results}

\subsection{Study Selection and Characteristics}

The search program yielded 147 references. After excluding 90 duplications, the remaining 57 abstracts were screened. Among them, we retrieved 11 articles for full-text review. Finally, three studies [11-13] fulfilling the inclusion criteria were included in this meta-analysis (Figure 1). All enrolled studies had the same principal investigator. All studies [11-13] were randomized, multicenter, and multinational studies designed to compare the clinical efficacy and safety of eravacycline with other comparators for adult patients with cIAI (Table 1). The inclusion criterion of these three studies was that adult patients had to have clinical evidence of cIAI requiring urgent surgical or percutaneous intervention within 48 hours of diagnosis. Two studies [11,13] compared eravacycline with ertapenem, and one [12] compared with meropenem. The test-of-cure evaluation was conducted 25 to 31 calendar days in two studies [11,12] and 10 to 14 days in one study [13] after the first dose of the study drug was administered for the patients with cIAI. The follow-up visit was performed 38 to 50 calendar days in one study [11] and 28 to 42 days in one study [13] after the first dose of study drug was administered. All of the domains in each study were classified as having a low risk of bias (Table 2).

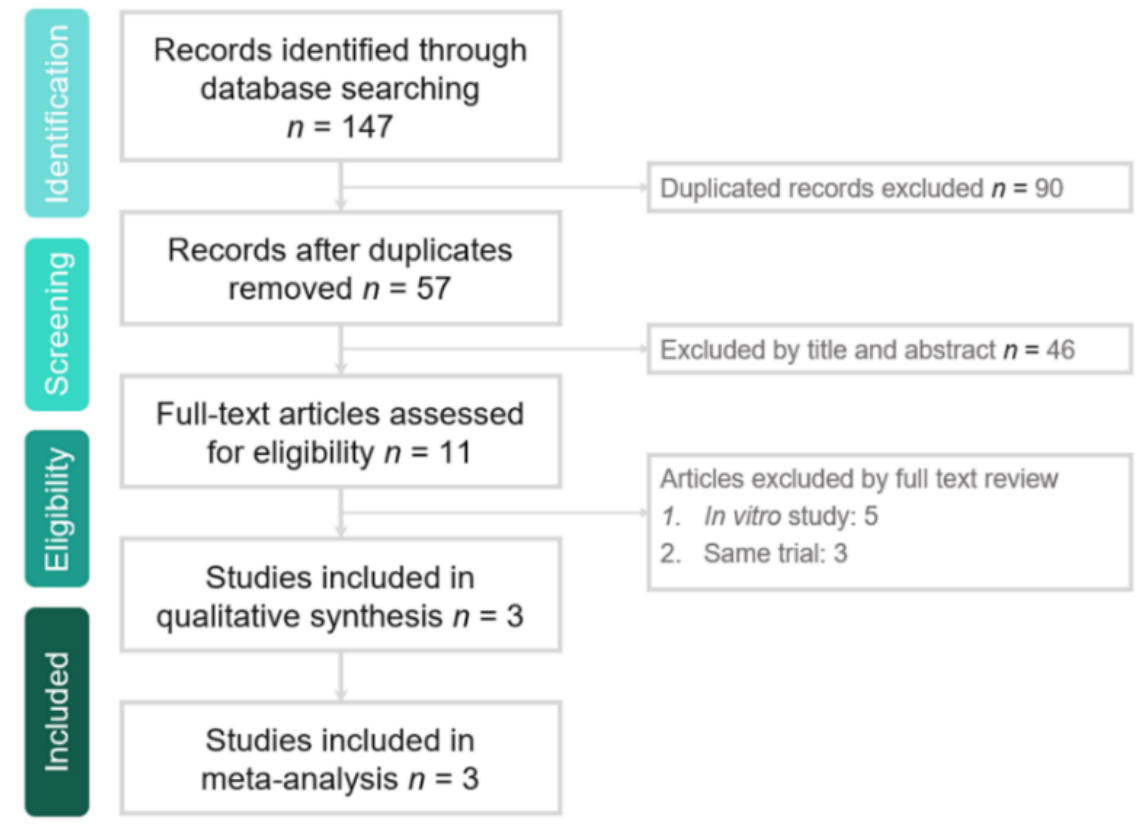

Figure 1. Study selection process flow. 
Table 1. Characteristics of included studies.

\begin{tabular}{|c|c|c|c|c|c|c|c|}
\hline \multirow{2}{*}{$\begin{array}{c}\text { Study, Published } \\
\text { Year }\end{array}$} & \multirow{2}{*}{ Study Design } & \multirow{2}{*}{ Study Site } & \multirow{2}{*}{ Study Period } & \multicolumn{2}{|c|}{ No. of Patients (ITT population) } & \multicolumn{2}{|c|}{ Dose Regimen } \\
\hline & & & & Eravacycline & Comparator & Eravacycline & Comparator \\
\hline $\begin{array}{l}\text { Solomkin et al, } \\
2014\end{array}$ & $\begin{array}{c}\text { Randomized, } \\
\text { double-blind trial }\end{array}$ & $\begin{array}{c}19 \text { sites in } 6 \\
\text { countries }\end{array}$ & 2011-2012 & $\begin{array}{c}56(1.5 \mathrm{mg} / \mathrm{kg}), 57 \\
(1.0 \mathrm{mg} / \mathrm{kg})\end{array}$ & 30 & $\begin{array}{c}1.5 \mathrm{mg} / \mathrm{kg} \text { or } 1.0 \\
\mathrm{mg} / \mathrm{kg} \mathrm{q} 24 \mathrm{~h}\end{array}$ & Ertapenem $1 \mathrm{~g} \mathrm{q} 24 \mathrm{~h}$ \\
\hline IGNITE1, 2017 & $\begin{array}{c}\text { Randomized, } \\
\text { double-blind trial }\end{array}$ & $\begin{array}{c}66 \text { sites in } 11 \\
\text { countries }\end{array}$ & 2013-2014 & 270 & 271 & $1.0 \mathrm{mg} / \mathrm{kg} \mathrm{q} 12 \mathrm{~h}$ & Ertapenem $1 \mathrm{~g}$ q24 h \\
\hline IGNITE4, 2018 & $\begin{array}{c}\text { Randomized, } \\
\text { double-blind trial }\end{array}$ & $\begin{array}{c}65 \text { sites in } 11 \\
\text { countries }\end{array}$ & 2016-2017 & 250 & 250 & $1.0 \mathrm{mg} / \mathrm{kg} \mathrm{q} 12 \mathrm{~h}$ & Meropenem $1 \mathrm{~g} \mathrm{q} 8 \mathrm{~h}$ \\
\hline
\end{tabular}

ITT, intention to treat; $q$, every; $h$, hour; $\mathrm{mg}$, milligram; $\mathrm{g}$, gram.

Table 2. Risk of bias per study and domain.

\begin{tabular}{cccc}
\hline \multirow{2}{*}{ Risk of Bias } & \multicolumn{3}{c}{ Study } \\
\cline { 2 - 4 } & IGNITE1, 2017 & IGNITE4, 2018 & Solomkin et al, 2014 \\
\hline Random sequence generation & low & low & low \\
Allocation concealment & low & low & low \\
Blinding of participants and personnel & low & low & low \\
Blinding of outcome assessment & low & low \\
Incomplete outcome data & low & low & low \\
Selective reporting & low & low \\
\hline
\end{tabular}




\subsection{Clinical Efficacy and Microbiologic Response}

Overall, eravacycline had a clinical cure rate $(88.7 \%, 559 / 630)$ at test-of-cure in modified intent-to-treat population similar to comparators $(90.1 \%, 492 / 546)$ in the treatment of cIAIs (risk ratio (RR), $0.99 ; 95 \% \mathrm{CI}, 0.95-1.03 ; I^{2}=0 \%$, Figure 2 ). In the microbiological intent-to-treat, clinically evaluable, and microbiological evaluable populations, no difference was found between eravacycline and comparators in terms of clinical cure rate at test-of-cure (Figure 2). In addition, no significant difference was observed between eravacycline and comparator in terms of clinical failure rate at test-of-cure in modified intent-to-treat population, microbiological intent-to-treat population, clinically evaluable population, and microbiological evaluable population (Figure 3).

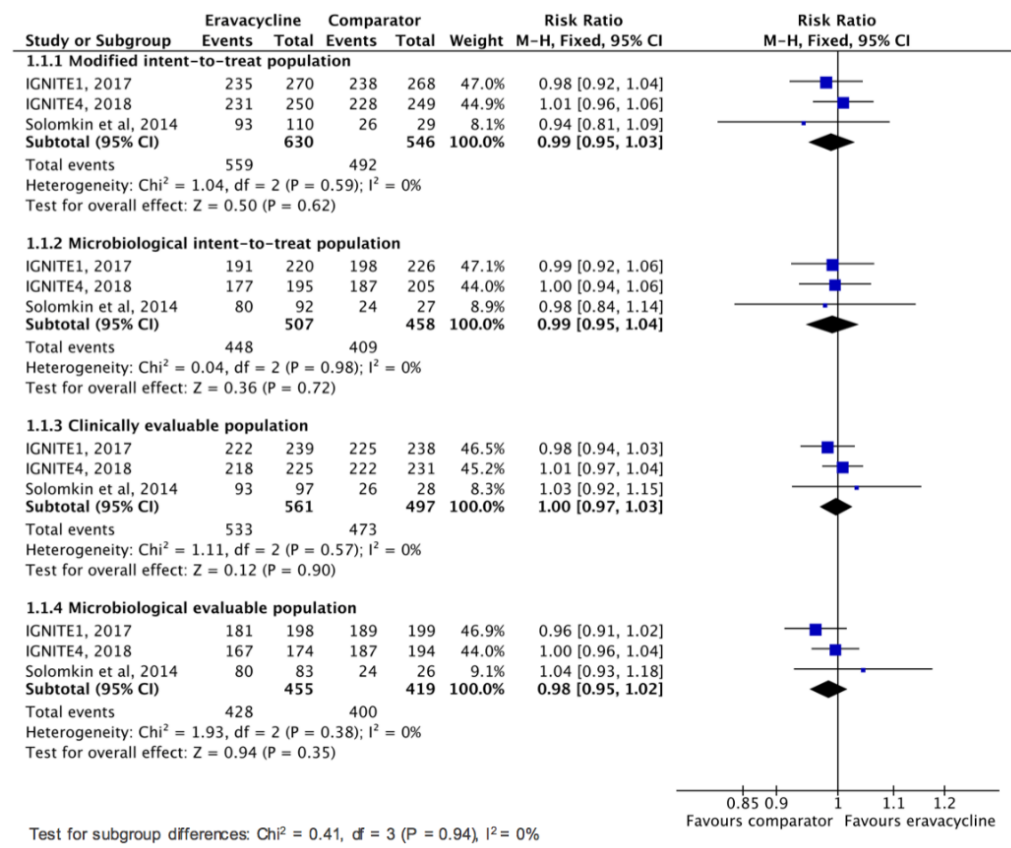

Figure 2. Overall clinical cure rates for eravacycline and comparators in the treatment of complicated intra-abdominal infections.

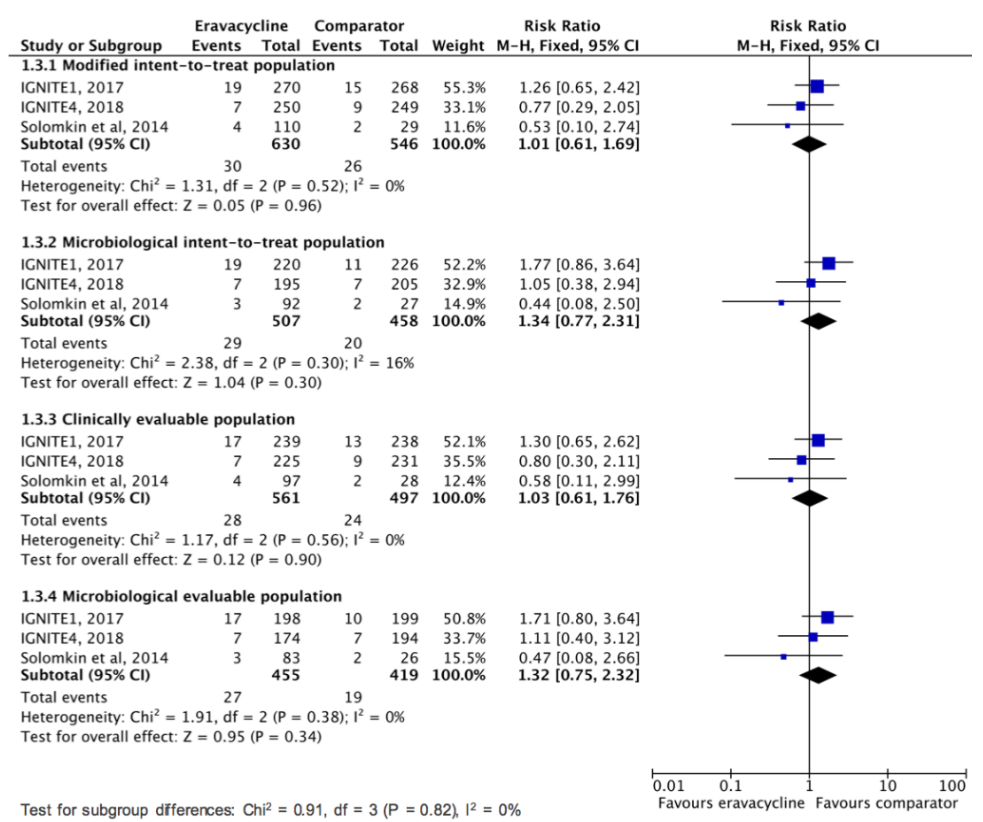

Figure 3. Overall clinical failure rates for eravacycline and comparators in the treatment of complicated intra-abdominal infections. 
Only two studies $[12,13]$ reported the outcome at end-of-treatment, and the pooled analysis showed no significant difference was observed between eravacycline and comparator in terms of clinical cure rate at end-of-treatment in modified intent-to-treat population (RR, $0.99 ; 95 \% \mathrm{CI}, 0.95-1.03 ; \mathrm{I}^{2}=1 \%$ ), microbiological intent-to-treat population ( $\mathrm{RR}, 0.98 ; 95 \% \mathrm{CI}, 0.94-1.03 ; I^{2}=0 \%$ ), clinically evaluable population (RR, $0.99 ; 95 \% \mathrm{CI}, 0.96-1.01 ; I^{2}=0 \%$ ), and microbiological evaluable population (RR, 0.98 ; $95 \%$ CI, $0.95-1.01 ; I^{2}=0 \%$ ). In addition, these two studies ${ }^{12,13}$ reported the outcome at follow-up, and the pooled analysis showed no significant difference was observed between eravacycline and comparator in terms of clinical cure rate at follow-up in modified intent-to-treat population (RR, 0.99; 95\% CI, 0.93-1.04; $I^{2}=0 \%$ ), microbiological intent-to-treat population (RR, 0.98; 95\% CI, 0.92-1.06; $I^{2}=0 \%$ ), clinically evaluable population (RR, $1.01 ; 95 \% \mathrm{CI}, 0.97-1.06 ; I^{2}=0 \%$ ), and microbiological evaluable population (RR, $1.02 ; 95 \% \mathrm{CI}, 0.97-1.07 ; I^{2}=30 \%$ ).

\subsection{Adverse Events}

In the pooled analysis of three studies reporting adverse events, we found that eravacycline was associated with a higher risk of treatment-emergent adverse events than comparators (Figure 4). However, no significant differences were found between eravacycline and comparators for the risk of serious adverse events, discontinuation of study drug because of adverse event, and all-cause mortality (Figure 4). The most common adverse event among the eravacycline group was nausea $(6.5 \%, 41 / 629)$ and vomiting $(3.8 \%, 24 / 629)$. Although the risks of nausea and vomiting in the eravacycline group were higher than those in the comparator group, these differences did not reach statistical significance (for nausea, RR, 4.79; 95\% CI, 0.84-27.14;7 $I^{2}=70 \%$, for vomiting, RR, 1.46 ; 95\% CI, $0.76-2.81 ; 7 \mathrm{I}^{2}=0 \%$ ).

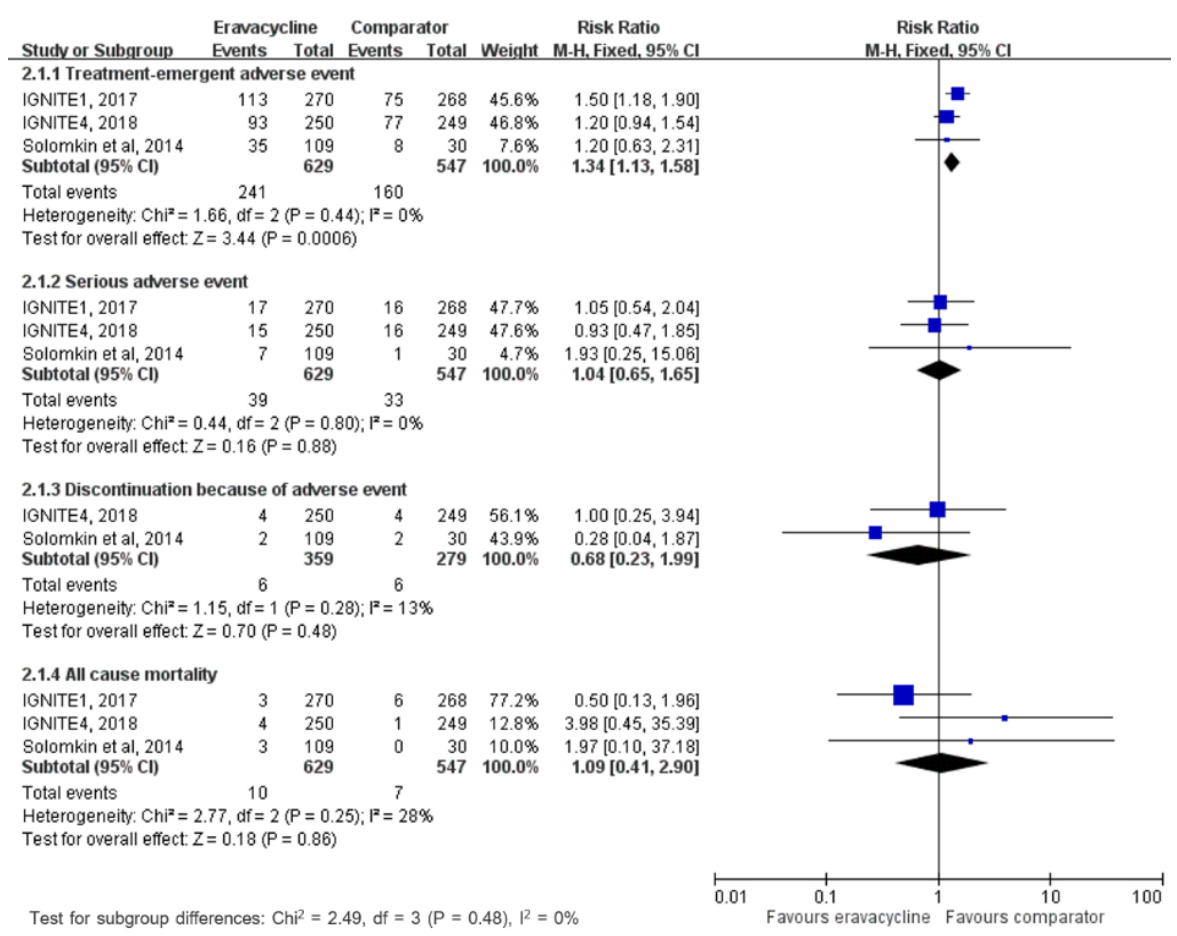

Figure 4. Adverse event risks with eravacycline and comparators in the treatment of complicated intra-abdominal infections.

\section{Discussion}

This first meta-analysis based on three RCTs [11-13] determined that the clinical efficacy of eravacycline is similar to that of other comparators in the treatment of adult patients with cIAIs. This significant finding is supported by the following analysis. First, the overall pooled clinical cure rate at test-of-cure of eravacycline in treating cIAIs was comparable to carbapenems in modified 
intent-to-treat, microbiological intent-to-treat, clinically evaluable, and microbiological evaluable populations. Second, pooled clinical failure rate at test-of-cure of eravacycline was as low as comparators in modified intent-to-treat, microbiological intent-to-treat, clinically evaluable, and microbiological evaluable populations. Third, this similarity in terms of clinical efficacy between eravacycline and comparators did not change with the timing of the outcome measure at end-of-treatment and follow-up. In summary, all of these findings indicated that eravacycline can be an effective therapeutic option in the treatment of adult patients with cIAIs.

The effectiveness of ceftaroline in the treatment of cIAIs in adult patients can be supported by in vitro studies $[7,9,10,15,16]$. In the surveillance of 2213 Gram-negative and 2423 Gram-positive pathogens in 13 Canadian hospitals, the minimum inhibitory concentration ${ }_{90}\left(\mathrm{MIC}_{90}\right.$ ) ranged from 0.5 to $2 \mu \mathrm{g} / \mathrm{mL}$ for 9 species of Enterobacteriaceae tested $(n=2067)$ and extended-spectrum $\beta$-lactamase producing E. coli $(n=141)$ and K. pneumoniae $(n=21)$ did not affect the potency of eravacycline in this study [10]. In another survey of more than 4000 Gram-negative pathogens in New York hospitals [7], eravacycline demonstrated great in vitro activity against Enterobacteriaceae-E. coli, K. pneumoniae, Enterobacter aerogenes, and Enterobacter cloacae with minimum inhibitory concentration ${ }_{50}$ $\left(\mathrm{MIC}_{50}\right) / \mathrm{MIC}_{90}$ of $0.12 / 0.5 \mu \mathrm{g} / \mathrm{mL}, 0.25 / 1 \mu \mathrm{g} / \mathrm{mL}, 0.25 / 1 \mu \mathrm{g} / \mathrm{mL}$, and $0.5 / 1 \mu \mathrm{g} / \mathrm{mL}$, respectively. Moreover, the potent activity was retained against multidrug-resistant (MDR) isolates, including carbapenem nonsusceptible strains [7,9]. In addition to aerobic bacteria, anerobic bacteria play important roles in the cIAIs. Eravacycline showed good in vitro activity against Bacteroides spp., Parabacteroides spp., and Clostridioides difficile (formerly Clostridium difficile) and eravacycline remained potent against the strains with tetracycline-specific resistance determinants and MDR anaerobic pathogens [15,16]. Overall, the potent in vitro activity of eravacycline against commonly encountered pathogens causing cIAI largely explains the great in vivo clinical response in this meta-analysis.

In addition to clinical efficacy of eravacycline for the treatment of cIAIs, we should consider the risk of adverse event while prescribing eravacycline. Nausea and vomiting were the most common adverse events, and the overall incidence of these adverse events were higher than those of comparators. Moreover, the pooled risk of treatment-emergent adverse events was higher in the eravacycline group than in the control group. These findings are consistent with previous pooled analysis of IGNITE1 and IGNITE4, in which eravacycline recipients had higher incidence of nausea ( $6.5 \mathrm{vs.} 0.6 \%$ ) and vomiting (3.7 vs. $2.5 \%$ ) [17]. In contrast, the incidence of serious adverse events, discontinuation of study drug because of adverse event, and all-cause mortality was similar between eravacycline and comparators. Therefore, the findings of this meta-analysis suggest that although eravacycline is associated with higher risk of mild adverse events than comparator, overall, eravacycline remains as safe as other comparators in the treatment of cIAI among adult patients.

This study has several limitations. First, only three RCTs were considered in this meta-analysis. Second, the usefulness of eravacycline in treating cIAIs was not assessed according to the disease severity. Third, we did not evaluate the correlation between in vitro activity and in vivo response of eravacycline against each specific pathogen, particularly antibiotic-resistant organisms, in this study.

\section{Conclusions}

In conclusion, eravacycline is as good as comparators in terms of efficacy and tolerance in the treatment of cIAI in adult patients.

Author Contributions: Conceptualization, S.-H.L., C.-C.L. and C.-M.C.; Data curation, S.-P.C., C.-C.L. and L.-C.L.; Formal analysis, S.-H.L., S.-P.C. and L.-C.L.; Writing—original draft, C.-C.L.; Writing-review \& editing, C.-M.C.

Conflicts of Interest: The authors declare no conflict of interest. 
Appendix A. Search Strategy

\begin{tabular}{|c|c|c|}
\hline \multicolumn{2}{|c|}{ PubMed Search Strategy_Last Searched on 26 May 2019} & \multirow{2}{*}{$\begin{array}{c}\text { Results } \\
74\end{array}$} \\
\hline 1 & $\begin{array}{l}\text { Eravacycline [Title/Abstract] OR TP-434 } \\
\text { [Title/Abstract] OR Xerava [Title/Abstract] }\end{array}$ & \\
\hline 2 & abdom* [Title/Abstract] & 330,674 \\
\hline 3 & 1 AND 2 & \\
\hline 4 & $\begin{array}{l}\text { Search (abdom* (Title/Abstract)) AND } \\
(((\text { Eravacycline (Title/Abstract)) OR TP-434 } \\
\text { (Title/Abstract)) OR Xerava (Title/Abstract)) }\end{array}$ & 22 \\
\hline \multicolumn{2}{|c|}{ Web of Science Search Strategy_Last Searched on 26 May 2019} & Results \\
\hline 1 & (Eravacycline) OR (Xerava) OR (TP-434) & 71 \\
\hline 2 & $($ abdom*) & 269,250 \\
\hline 3 & 1 AND 2 & \\
\hline 4 & \#1 AND \#2 & 20 \\
\hline \multicolumn{2}{|c|}{ EBSCO Search Strategy_Last Searched on 26 May 2019} & Results \\
\hline 1 & AB Eravacycline OR AB Xerava OR AB TP-434 & 176 \\
\hline 2 & $\mathrm{AB}$ abdom* & 495,125 \\
\hline 3 & 1 AND 2 & \\
\hline 4 & S1 AND S2 & 40 \\
\hline \multicolumn{2}{|c|}{ Cochrane Library Search Strategy_Last Searched on 26 May 2019} & Results \\
\hline 1 & $\begin{array}{l}\text { (Eravacycline):ti,ab,kw OR (TP-434):ti,ab,kw OR } \\
\text { (Xerava):ti,ab,kw }\end{array}$ & 12 \\
\hline 2 & $($ abdom*):ti,ab,kw & 40,365 \\
\hline 3 & 1 AND 2 & \\
\hline 4 & \#1 AND \#2 & 5 \\
\hline \multicolumn{2}{|c|}{ Ovid Medline Search Strategy_Last Searched on 26 May 2019} & Results \\
\hline 1 & (Eravacycline or Xerava or TP-434).ab. & 82 \\
\hline 2 & abdom*.ab & 373,974 \\
\hline 3 & 1 AND 2 & \\
\hline 4 & 1 and 2 & 24 \\
\hline \multicolumn{2}{|c|}{ Embase Search Strategy-Last Searched on 26 May 2019} & Results \\
\hline 1 & $\begin{array}{l}\text { eravacycline:ti,ab,kw OR xerava:ti,ab,kw OR 'tp } \\
434^{\prime} \text { :ti,ab,kw }\end{array}$ & 87 \\
\hline 2 & abdom*:ti,ab,kw & 508,670 \\
\hline 3 & 1 AND 2 & \\
\hline 4 & \#1 AND \#2 & 27 \\
\hline \multicolumn{2}{|c|}{ ClinicalTrials.gov Search Strategy-Last Searched on May 26, 2019} & Results \\
\hline 1 & Eravacycline (completed studies) & 9 \\
\hline
\end{tabular}

\section{References}

1. Solomkin, J.S.; Mazuski, J.E.; Baron, E.J.; Sawyer, R.G.; Nathens, A.B.; DiPiro, J.T.; Buchman, T.; Dellinger, E.P.; Jernigan, J.; Gorbach, S.; et al. Guidelines for the selection of anti-infective agents for complicated intra-abdominal infections. Clin. Infect. Dis. 2003, 37, 997-1005. [CrossRef] [PubMed]

2. Solomkin, J.S.; Mazuski, J.E.; Bradley, J.S.; Rodvold, K.A.; Goldstein, E.J.; Baron, E.J.; O’Neill, P.J.; Chow, A.W.; Dellinger, E.P.; Eachempati, S.R.; et al. Diagnosis and management of complicated intra-abdominal infection in adults and children: guidelines by the Surgical Infection Society and the Infectious Diseases Society of America. Clin. Infect. Dis. 2010, 50, 133-164. [CrossRef] [PubMed] 
3. Brink, A.J.; Botha, R.F.; Poswa, X.; Senekal, M.; Badal, R.E.; Grolman, D.C.; Richards, G.A.; Feldman, C.; Boffard, K.D.; Veller, M.; et al. Antimicrobial susceptibility of Gram-negative pathogens isolated from patients with complicated intra-abdominal infections in South African hospitals (SMART Study 2004-2009): Impact of the new carbapenem breakpoints. Surg. Infect. (Larchmt) 2012, 13, 43-49. [CrossRef] [PubMed]

4. Lee, Y.L.; Chen, Y.S.; Toh, H.S.; Huang, C.C.; Liu, Y.M.; Ho, C.M.; Lu, P.L.; Ko, W.C.; Chen, Y.H.; Wang, J.H.; et al. Antimicrobial susceptibility of pathogens isolated from patients with complicated intra-abdominal infections at five medical centers in Taiwan that continuously participated in the Study for Monitoring Antimicrobial Resistance Trends (SMART) from 2006 to 2010. Int. J. Antimicrob. Agents 2012, 40, S29-S36. [PubMed]

5. Sheng, W.H.; Badal, R.E.; Hsueh, P.R. Distribution of extended-spectrum beta-lactamases, AmpC beta-lactamases, and carbapenemases among Enterobacteriaceae isolates causing intra-abdominal infections in the Asia-Pacific region: Results of the study for Monitoring Antimicrobial Resistance Trends (SMART). Antimicrob. Agents Chemother. 2013, 57, 2981-2988. [PubMed]

6. Zhanel, G.G.; Cheung, D.; Adam, H.; Zelenitsky, S.; Golden, A.; Schweizer, F.; Gorityala, B.; Lagacé-Wiens, P.R.; Walkty, A.; Gin, A.S.; et al. Review of Eravacycline, a Novel Fluorocycline Antibacterial Agent. Drugs 2016, 76, 567-588. [CrossRef] [PubMed]

7. Abdallah, M.; Olafisoye, O.; Cortes, C.; Urban, C.; Landman, D.; Quale, J. Activity of eravacycline against Enterobacteriaceae and Acinetobacter baumannii, including multidrug-resistant isolates, from New York City. Antimicrob. Agents Chemother. 2015, 59, 1802-1805. [CrossRef] [PubMed]

8. Livermore, D.M.; Mushtaq, S.; Warner, M.; Woodford, N. In vitro activity of eravacycline against Carbapenem-Resistant Enterobacteriaceae and Acinetobacter baumannii. Antimicrob. Agents Chemother. 2016, 60, 3840-3844. [CrossRef] [PubMed]

9. Seifert, H.; Stefanik, D.; Sutcliffe, J.A.; Higgins, P.G. In-vitro activity of the novel fluorocycline eravacycline against carbapenem non-susceptible Acinetobacter baumannii. Int. J. Antimicrob. Agents 2018, 51, 62-64. [CrossRef] [PubMed]

10. Zhanel, G.G.; Baxter, M.R.; Adam, H.J.; Sutcliffe, J.; Karlowsky, J.A. In vitro activity of eravacycline against 2213 Gram-negative and 2424 Gram-positive bacterial pathogens isolated in Canadian hospital laboratories: CANWARD surveillance study 2014-2015. Diagn. Microbiol. Infect. Dis. 2018, 91, 55-62. [CrossRef] [PubMed]

11. Solomkin, J.; Evans, D.; Slepavicius, A.; Lee, P.; Marsh, A.; Tsai, L.; Sutcliffe, J.A.; Horn, P.A. Assessing the efficacy and safety of eravacycline vs ertapenem in complicated intra-abdominal infections in the Investigating Gram-Negative Infections Treated with Eravacycline (IGNITE 1) trial: A randomized clinical trial. JAMA Surg. 2017, 152, 224-232. [CrossRef] [PubMed]

12. Solomkin, J.S.; Gardovskis, J.; Lawrence, K.; Montravers, P.; Sway, A.; Evans, D.; Tsai, L. IGNITE4: Results of a phase 3 , randomized, multicenter, prospective trial of eravacycline vs. meropenem in the treatment of complicated intra-abdominal infections. Clin. Infect. Dis. 2018. [CrossRef] [PubMed]

13. Solomkin, J.S.; Ramesh, M.K.; Cesnauskas, G.; Novikovs, N.; Stefanova, P.; Sutcliffe, J.A.; Walpole, S.M.; Horn, P.T. Phase 2, randomized, double-blind study of the efficacy and safety of two dose regimens of eravacycline versus ertapenem for adult community-acquired complicated intra-abdominal infections. Antimicrob. Agents Chemother. 2014, 58, 1847-1854. [CrossRef]

14. Higgins, J.P.; Altman, D.G.; Gotzsche, P.C.; Juni, P.; Moher, D.; Oxman, A.D.; Savovic, J.; Schulz, K.F.; Weeks, L.; Sterne, J.A. The Cochrane Collaboration's tool for assessing risk of bias in randomised trials. BMJ 2011, 343, d5928. [CrossRef]

15. Goldstein, E.J.C.; Citron, D.M.; Tyrrell, K.L. In vitro activity of eravacycline and comparator antimicrobials against 143 recent strains of Bacteroides and Parabacteroides species. Anaerobe 2018, 52, 122-124. [CrossRef]

16. Snydman, D.R.; McDermott, L.A.; Jacobus, N.V.; Kerstein, K.; Grossman, T.H.; Sutcliffe, J.A. Evaluation of the in vitro activity of eravacycline against a broad spectrum of recent clinical anaerobic isolates. Antimicrob. Agents Chemother. 2018, 62, e02206-17. [CrossRef]

17. Tetraphase Pharmaceuticals Inc. Xerava (Eravacycline): US Prescribing Information. 2018. Available online: http://www.fda.gov (accessed on 23 May 2019).

(C) 2019 by the authors. Licensee MDPI, Basel, Switzerland. This article is an open access article distributed under the terms and conditions of the Creative Commons Attribution (CC BY) license (http://creativecommons.org/licenses/by/4.0/). 